Research article

Open Access

\title{
Broad-range PCR, cloning and sequencing of the full 16S rRNA gene for detection of bacterial DNA in synovial fluid samples of Tunisian patients with reactive and undifferentiated arthritis
}

\author{
Mariam Siala ${ }^{1}$, Radhouane Gdoura ${ }^{1}$, Hela Fourati ${ }^{2}$, Markus Rihl${ }^{3}$, Benoit Jaulhac ${ }^{4}$, \\ Mohamed Younes ${ }^{5}$, Jean Sibilia4, Sofien Baklouti2 ${ }^{2}$ Naceur Bargaoui ${ }^{5}$, Slaheddine Sellami6, \\ Abdelghani Sghir 7,8 and Adnane Hammami ${ }^{1}$
}

\author{
'Laboratoire de Recherche 'Micro-organismes et Pathologie Humaine', EPS Habib Bourguiba, Rue El Ferdaous, 3029 Sfax, Tunisie \\ ${ }^{2}$ Service de Rhumatologie Hôpital Hedi Chaker, Avenue Majida Boulila, 3029 Sfax, Tunisie \\ ${ }^{3}$ Hannover Medical School (MHH), Clinic for Immunology and Rheumatology, 30625 Hannover; Germany \\ ${ }^{4}$ Laboratoire de Physiopathologie des Interactions Hôte-bactérie, UPRES-EA 3432, Faculté de Médecine, Université Louis-Pasteur, rue Koeberlé, \\ 67000 Strasbourg, France \\ ${ }^{5}$ Service de Rhumatologie, EPS Fattouma Bourguiba, Rue 1er Juin, 5019 Monastir, Tunisie \\ 6Service de Rhumatologie, EPS La Rabta, rue 7051 Centre Urbain Nord, 1082 Tunis, Tunisie \\ ${ }^{7}$ CNRS-UMR 8030, CEA-Genoscope, rue Gaston Crémieux, 91000 Évry, France \\ 8University of Evry Val d'Essonne, Boulevard François Mitterrand, 91025 Évry Cedex, 91000 Évry, France
}

Corresponding author: Adnane Hammami, adnene.hammami@rns.tn

Received: 8 Apr 2009 Revisions requested: 21 May 2009 Revisions received: 29 May 2009 Accepted: 1 Jul 2009 Published: 1 Jul 2009

Arthritis Research \& Therapy 2009, 11:R102 (doi:10.1186/ar2748)

This article is online at: http://arthritis-research.com/content/11/4/R102

(c) 2009 Siala et al.; licensee BioMed Central Ltd.

This is an open access article distributed under the terms of the Creative Commons Attribution License (http://creativecommons.org/licenses/by/2.0), which permits unrestricted use, distribution, and reproduction in any medium, provided the original work is properly cited.

\begin{abstract}
Introduction Broad-range rDNA PCR provides an alternative, cultivation-independent approach for identifying bacterial DNA in reactive and other form of arthritis. The aim of this study was to use broad-range rDNA PCR targeting the 16S rRNA gene in patients with reactive and other forms of arthritis and to screen for the presence of DNA from any given bacterial species in synovial fluid (SF) samples.

Methods We examined the SF samples from a total of 27 patients consisting of patients with reactive arthritis $(\operatorname{ReA})(n=$ $5)$, undifferentiated arthritis (UA) $(n=9)$, rheumatoid arthritis ( $n$ $=7)$, and osteoarthritis $(n=6)$ of which the latter two were used as controls. Using broad-range bacterial PCR amplifying a 1400 bp fragment from the 16S rRNA gene, we identified and sequenced at least 24 clones from each SF sample. To identify the corresponding bacteria, DNA sequences were compared to the EMBL (European Molecular Biology Laboratory) database.

Results Bacterial DNA was identified in 20 of the 27 SF samples $(74,10 \%)$. Analysis of a large number of sequences revealed the presence of DNA from more than one single

bacterial species in the SF of all patients studied. The nearly complete sequences of the $1400 \mathrm{bp}$ were obtained for most of the detected species. DNA of bacterial species including Shigella species, Escherichia species, and other coli-form bacteria as well as opportunistic pathogens such as Stenotrophomonas maltophilia and Achromobacter xylosoxidans were shared in all arthritis patients. Among pathogens described to trigger ReA, DNA from Shigella sonnei was found in ReA and UA patients. We also detected DNA from rarely occurring human pathogens such as Aranicola species and Pantoea ananatis. We also found DNA from bacteria so far not described in human infections such as Bacillus niacini, Paenibacillus humicus, Diaphorobacter species and uncultured bacterium genera incertae sedis OP10.

Conclusions Broad-range PCR followed by cloning and sequencing the entire 16S rDNA, allowed the identification of the bacterial DNA environment in the SF samples of arthritic patients. We found a wide spectrum of bacteria including those known to be involved in $\operatorname{ReA}$ and others not previously associated with arthritis.
\end{abstract}




\section{Introduction}

The actual pathogenic event initiating arthritis is largely unknown. For several forms of arthritis, an infectious etiology has been postulated [1-3]. In particular, reactive arthritis $(\operatorname{Re} A)$ is known to be triggered by a variety of bacteria. For Salmonella, Yersinia, and Chlamydia, a persistent infection has been hypothesized due to the intraarticular presence of bacterial antigens, DNA, and/or RNA [4-6]. There is also evidence that undifferentiated arthritis is a form of $\operatorname{ReA}$ ('forme fruste') possibly due to a preceding but asymptomatic infection $[7,8]$. PCR using universal 16S rRNA primers is a highly sensitive tool allowing detection of unknown, that is unsuspected, pathogens relating to all eubacterial species [9-11]. This tool has been used before in patients with ReA, undifferentiated arthritis (UA), and other arthropathies. However, in most studies, the PCR products were of sufficient length to determine the genus of the bacteria in the synovial samples, but were not long enough to identify the species level [12-14].

We previously used the broad-range PCR, cloning and sequencing the entire $16 \mathrm{~S}$ rDNA and demonstrated the presence of a large number of bacterial DNA in the synovial tissue (ST) of patients with ReA, UA, and other arthropathies [15]. These bacterial DNA were mainly derived from commensals that are normally present in the skin and gut. We also detected DNA of specific bacterial groups that have not been detected in arthritis samples or in human infections so far, suggesting that these new bacteria possibly could have a pathogenic relevance, particularly with regard to the ST. The detection of such a variety of bacterial groups after cloning and near fulllength 16S rDNA sequencing obtained in ST samples has raised the question if the identical bacterial DNA communities could reside in both the ST and the synovial fluid (SF) of matched arthritis patients [15]. In addition, the composition of bacterial DNA from ST samples has not been compared comprehensively with that of matched SF samples in arthritis patients. Besides, a detailed analysis of SF bacterial DNA and their comparison with those from corresponding ST samples might help to determine whether intraarticular bacterial DNA might change over time between the two synovial compartments.

As opposed to SF $[12,16]$, the bacterial DNA communities in the ST are well documented [13,14,17-19] and to our knowledge, there is no study that has amplified the entire 16S rDNA from SF samples.

Accordingly, we now continue our study using PCR as well as cloning and sequencing of the entire $16 \mathrm{~S}$ rDNA to identify any bacterial DNA potentially present in the SF samples of patients with $\mathrm{ReA}, \mathrm{UA}$, and other forms of arthritis who were also analyzed previously [15].

\section{Materials and methods \\ Patients and synovial fluid samples}

Twenty-seven patients with active arthritis and knee effusion gave informed consent and were included in the study, which was approved by the local ethical committees. ST samples of these patients were used in a previous study [15]. The clinical characteristics as well as technical aspects regarding prevention of contamination during sampling have recently been published in detail [15].

Among the 27 patients included in the study, five were diagnosed with $\operatorname{ReA}$, and nine with undifferentiated arthritis (UA); we also included seven patients with rheumatoid arthritis (RA) and six with osteoarthritis (OA) who served as controls. In the current study, the 27 patients were identical to the 27 patients analyzed in our previously published study (from patient 2 to patient 28) [15]. The clinical features and demographic characteristics of the patients are summarized in Table 1. SF samples were aspirated by standard needle puncture, snap frozen in liquid nitrogen, and stored at $-80^{\circ} \mathrm{C}$ until analysis.

\section{Automated DNA extraction from synovial fluid and broad-range PCR amplification of 16S rRNA genes, cloning and sequencing of bacterial DNA}

For 10 minutes, $500 \mu \mathrm{l}$ of SF were centrifuged at $10,000 \mathrm{~g}$. The whole SF cell pellet was subjected to DNA extraction using the MagNA Pure system (Roche Molecular Biochemicals, Meylan, France). Prior to the MagNA Pure extraction, 500 $\mu$ l of lysis buffer (200 mM sodium chloride, $20 \mathrm{mM}$ Tris hydrochloric acid, $\mathrm{pH} 8,50 \mathrm{mM}$ ethylenediaminetetraacetic acid and $1 \% \mathrm{SDS}$ ) and $25 \mu \mathrm{l}$ of proteinase $\mathrm{K}(10 \mathrm{mg} / \mathrm{ml})$ (Sigma, St Louis, MO, USA) were added to the SF cell pellet.

Bacterial 16S rDNA fragments were amplified from SF extracted DNA by broad-range PCR amplification using universal bacterial 16S rRNA gene-specific oligonucleotide primers Bac08 and Uni1390, as previously described [15]. Products of the expected size (approximately $1400 \mathrm{bp}$ ) were inserted into a vector using a cloning kit (pGEM-T vector; Promega, Madison, WI, USA). Sequencing and 16S rDNA analysis were performed as previously described [15].

\section{Data analysis}

Statistical analysis was performed using SPSS 11.0 software (SPSS; Chicago, IL, USA). A $P<0.05$ was considered statistically significant.

\section{Results PCR positivity by the broad-range PCR amplification system}

PCR was positive in 20 of the 27 (74.10\%) patients indicating the presence of bacterial 16S rDNA within the synovial samples. Of note, PCR was positive in five out of five (100\%) patients with $\operatorname{ReA}$ and also in nine out of nine (100\%) patients with UA. In the control group, bacterial 16S rDNA was ampli- 
Table 1

\begin{tabular}{|c|c|c|c|c|}
\hline Diagnosis (patients; $\mathrm{n}=27$ ) & $\begin{array}{l}\text { Median disease duration, } \\
\text { months (range) }\end{array}$ & $\begin{array}{l}\text { Actual age or median age, } \\
\text { years (range) }\end{array}$ & Sex ratio $(M / F)$ & Clinical details \\
\hline $\operatorname{ReA}(n=5)$ & $2.4(1$ to 6$)$ & 32.4 (20 to 50$)$ & $4: 1$ & \\
\hline 1 & & 22 & M & $\begin{array}{l}\text { Sexually acquired } \mathrm{ReA} ; \mathrm{C} t \lg \mathrm{g} \\
\text { positive }^{\mathrm{a}}, \mathrm{Ct} \lg \mathrm{A} \text { positive } \\
\text { serology }^{\mathrm{b}}\end{array}$ \\
\hline 2 & & 40 & $\mathrm{~F}$ & Sexually acquired $\operatorname{ReA}$; \\
\hline 3 & & 20 & M & $\begin{array}{l}\text { Sexually acquired } \mathrm{ReA} ; \mathrm{C} t \lg \mathrm{Ig} \\
\text { positive serologya; Ct lgA } \\
\text { positive serologyb; B27+d }\end{array}$ \\
\hline 4 & & 50 & M & $\begin{array}{l}\text { Sexually acquired } \mathrm{ReA} ; \mathrm{Ct} \lg \mathrm{A} \\
\text { positive serologyb; Ct positive } \\
\mathrm{PCR}^{\mathrm{c}} ; \mathrm{B} 27+\mathrm{d}\end{array}$ \\
\hline 5 & & 30 & M & Sexually acquired $\operatorname{Re} A$ \\
\hline $\mathrm{UA}(\mathrm{n}=9)$ & 25 ( 2 to 60$)$ & 40 (22 to 59$)$ & $5: 4$ & - \\
\hline $\mathrm{RA}(n=7)$ & 66 (12 to 228 ) & 44 (39 to 53 ) & $2: 5$ & - \\
\hline OA $(n=6)$ & $14(12$ to 24$)$ & 58 (44 to 70$)$ & $5: 1$ & - \\
\hline
\end{tabular}

a Serology was determined for Ct-lgG antibodies by Micro Immunofluorescence assay as described by Wang and Grayston [29]. bSerology was determined for Ct-lgA antibodies by ELISA (Labsystems, Hilsinki, Finland). ${ }^{\circ}$ Chlamydia PCR in genital swabs was determined by Cobas Amplicor PCR assay (Roche Diagnostics Molecular Systems, Inc, CA, USA). dHLA-B27 positivity was determined using a microcytotoxicity assay. $\mathrm{C} t=\mathrm{Chlamydia}$ trachomatis; $\mathrm{RA}=$ rheumatoid arthritis; $\mathrm{ReA}=$ reactive arthritis; $\mathrm{OA}=$ osteoarthritis; $\mathrm{UA}=$ undifferentiated arthritis.

fied in three out of seven (43\%) RA patients and in three of six (50\%) OA patients. Accordingly, the presence of bacterial DNA within the SF samples was significantly higher in $\operatorname{ReA}$ and UA patients as compared with RA and OA patients (14 out of $14(100 \%)$ vs 6 of $13(46.2 \%) ; P=0.006)$.

At least 24 individual clones were selected from each PCR product and sequenced. A summary of the total number of sequences analyzed in each patient is depicted in Table 2. Only full-length (1300 to $1400 \mathrm{bp}$ ) sequences of high quality were analyzed in detail. Most bacterial sequences had at least $97 \%$ sequence similarity with any known cultivated or uncultured bacteria. The percentage of similarity to the best fit sequence in the database, the accession number, and the sequence length of each probe are listed in Table 3.

\section{Bacterial 16S rDNA sequences identified in synovial fluid samples}

Each patient's SF samples contained a diverse range of bacterial DNA-related species (Table 2). The majority of pathogens across all groups were identified as Shigella species and Stenotrophomonas maltophilia. DNA from a total of 69 various individual bacterial species were detected in SF samples from ReA and UA patients, whereas only 10 different bacterial DNA were found in SF samples from RA and OA patients. In addition, DNA from 20 bacterial species was detected in both the study and the control groups.

In ReA and UA patients, apart from Shigella sonnei DNA, there was no other DNA-derived bacteria so far described to trigger ReA. There were sequences from commensal bacteria, in particular those from the skin or the intestinal tract (Propionibacterium acnes, Escherichia coli, and other coliform bacteria). We also detected additional species previously assigned to the Pseudomonas genus such as DNA of Pseudomonas poae, Delftia acidovorans, and Burkholderia cepacia. A detailed sequence analysis of the PCR-positive samples of ReA and UA patients revealed a number of DNA of bacteria that have previously been described in human infections but not in arthritis, including Rhizobium radiobacter, Pantoea ananatis, and Capnocytophaga sputigena. Except for $P$. ananatis, these DNA sequences were observed in only one individual (Table 3)

DNA products from environmental bacteria previously detected in arthritis, such as Achromobacter xylosoxidans, Alcaligenes faecalis, and Flavobacterium mizutaii were commonly found in both the study and control groups. DNA of Aranicola species bacterium rarely described in humans but not associated with arthritis was common in patients with $\operatorname{ReA}$, $\mathrm{UA}$, and RA and was detected in more than one case (Table 3).

Some clones fell into environmental species not previously reported in human infections including DNA of Paenibacillus humicus, Bacillus niacini, and Diaphorobacter species, and other bacteria, which have not yet been cultured (Table 3).

DNA from the candidate division OP10 bacterium was detected in SF samples of only two patients with UA. These 
Arthritis Research \& Therapy Vol 11 No 4 Siala et al.

Table 2

Details of bacterial species-derived DNA sequences identified in each patient ${ }^{\star}$

Patient Total number of bacterial DNA sequences DNA sequences identified in each patient

$\operatorname{Re} A$

145

2

47

3

UA

$10 \times$ Stenotrophomonas maltophilia, $7 \times$ Shigella species, $6 \times$ Propionibacterium acnes, 3 $\times$ Ralstonia species, $3 \times$ Shigella sonnei, $3 \times$ uncultured bacterium, $2 \times$ Bradyrhizobium elkanii, $1 \times$ uncultured $\gamma$ proteobacterium, $1 \times$ uncultured $\gamma$ proteobacterium, $2 \times$ uncultured Sphingobacterium species, $1 \times$ Aranicola species, $1 \times$ Agrobacterium species, $1 \times$ Burkholderia species, $1 \times$ Escherichia coli, $1 \times$ Paenibacillus humicus, $1 \times$ Pantoea ananatis, $1 \times$ uncultured $\beta$ proteobacterium.

$13 \times$ Stenotrophomonas maltophilia, $4 \times$ Shigella species, $3 \times$ Aranicola species, $3 \times$ Ralstonia species, $2 \times \beta$ proteobacterium, $2 \times \gamma$ proteobacterium, $2 \times$ uncultured $\beta$ proteobacterium, $1 \times$ Agrobacterium species, $1 \times$ Bacteroidetes bacterium, $1 \times$ Escherichia coli, $1 \times$ Escherichia species, $1 \times$ Flavobacterium mizutaii, $1 \times$ Pantoea ananatis, $1 \times$ Paenibacillus humicus, $1 \times$ Paenibacillus species, $1 \times$ Propionibacterium acnes, $3 \times$ Shigella sonnei $1 \times$ Streptococcus mitis, $1 \times$ swine manure bacterium, $1 \times$ uncultured $\alpha$ proteobacterium, $1 \times$ uncultured Bacteroidetes bacterium, $1 \times$ uncultured Flavobacterium species, $1 \times$ uncultured soil bacterium.

$10 \times$ Shigella species, $9 \times$ Stenotrophomonas maltophilia, $3 \times$ Aranicola species, $3 \times$ uncultured bacterium,, $2 \times$ Escherichia species, $2 \times$ Paenibacillus humicus, $2 \times$ Streptococcus mitis, $1 \times$ Achromobacter xylosoxidans, $1 \times$ Bradyrhizobium elkanii, $1 \times$ Bacteroidetes bacterium, $1 \times$ Flavobacterium mizutaii, $1 \times$ p proteobacterium, $1 \times$ Propionibacterium acnes, $1 \times$ Shigella sonnei, $1 \times$ Streptococcus mitis, $1 \times$ uncultured Flavobacterium species, $1 \times$ uncultured Methylococcaceae bacterium, $1 \times$ uncultured Bacteroidetes bacterium

$11 \times$ Stenotrophomonas maltophilia, $6 \times \gamma$ proteobacterium, $5 \times$ Shigella species, $5 \times$ Shigella sonnei, $3 \times$ Aranicola species, $3 \times$ Comamonas testosteroni, $3 \times$ uncultured bacterium, $2 \times$ Escherichia species, $2 \times$ Propionibacterium acnes, $2 \times$ uncultured Flavobacterium species, $2 \times$ uncultured $\beta$ proteobacterium, $1 \times$ Alcaligenes faecalis, $1 \times$ Flavobacterium mizutaii, $1 \times$ Paenibacillus humicus $1 \times$ Pelomonas saccharophila, $1 \times$ Pseudomonas species.

$5 \times$ Escherichia coli, $4 \times$ Stenotrophomonas maltophilia, $3 \times$ Escherichia coli, $2 \times$ Pseudomonas species, $2 \times$ Shigella species, $2 \times$ Sphingomonas faeni, $1 \times$ Alcaligenes faecalis, $1 \times \beta$ proteobacterium, $1 \times$ Comamonas testosteroni, $1 \times$ Diaphorobacter species, $1 \times$ Delftia acidovorans, $1 \times$ Flavobacterium mizutaii, $1 \times \gamma$ proteobacterium, $1 \times$ uncultured bacterium, $1 \times$ uncultured $\beta$ proteobacterium, $1 \times$ Pantoea ananatis, $1 \times$ Propionibacterium acnes, $1 \times$ Ralstonia species, $1 \times$ Shigella sonnei,

$13 \times$ Stenotrophomonas maltophilia, $9 \times$ Shigella species, $7 \times \gamma$ proteobacterium, $2 \times$ uncultured bacterium, $2 \times$ uncultured Veillonella species, $1 \times$ Alcaligenes faecalis, $1 \times$ Agrobacterium species, $1 \times$ Escherichia species, $1 \times$ Streptococcus thermophilus, $1 \times$ Flavobacterium mizutaii, $1 \times$ Propionibacterium acnes, $1 \times$ uncultured $\beta$ proteobacterium, $1 \times$ uncultured bacterium, $1 \times$ uncultured candidate division OP10 bacterium, $1 \times$ Pantoea ananatis, $1 \times$ Shigella sonnei, $1 \times$ unidentiefied bacterium, $1 \times$ uncultured Flavobacterium species, $1 \times$ uncultured Sphingobacterium species.

$8 \times$ Stenotrophomonas maltophilia, $3 \times$ Shigella species, $2 \times$ Comamonas testosteroni, 2 $\times$ Paenibacillus humicus, $2 \times$ Ralstonia species, $2 \times$ uncultured bacterium, $2 \times$ uncultured $\beta$ proteobacterium, $2 \times$ uncultured Flavobacterium species, $1 \times$ Burkholderia species, $1 \times$ Bradyrhizobium elkanii, $1 \times$ Bacteroidetes bacterium, $1 \times$ Escherichia coli, $1 \times$ Flavobacterium mizutaii, $1 \times \gamma$ proteobacterium, $1 \times$ Shigella sonnei

$15 \times$ Stenotrophomonas maltophilia, $5 \times$ Shigella species, $3 \times$ Escherichia species, $3 \times$ Paenibacillus humicus, $2 \times$ uncultured bacterium, $2 \times$ uncultured $\beta$ proteobacterium, $1 \times$ Alcaligenes faecalis, $1 \times$ Aranicola species, $1 \times$ Bradyrhizobium elkanii, $1 \times$ Flavobacterium mizutaii, $1 \times \gamma$ proteobacterium, $1 \times$ Ralstonia species, $1 \times$ Staphylococcus pasteuri, $1 \times$ Streptococcus mitis, $1 \times$ Streptococcus pneumoniae, $1 \times$ Streptococcus species, $1 \times$ uncultured candidate division OP10 bacterium, $1 \times$ uncultured Streptococcus species, $1 \times$ uncultured Veillonella species.

$11 \times$ Aquabacterium commune, $8 \times$ Shigella species, $3 \times$ Aranicola species, $3 \times$ Paenibacillus humicus, $2 \times$ Alcaligenes faecalis, 2 uncultured bacterium, $1 \times \beta$ proteobacterium, $1 \times$ Comamonas testosteroni, $1 \times$ Escherichia species, $1 \times$ Kocuria species, $1 \times$ Pseudomonas species, $1 \times$ Paracoccus species, $1 \times$ Streptococcus thermophilus, $1 \times$ Streptococcus species, $1 \times$ uncultured $\beta$ proteobacterium 
$10 \quad 41$

$11 \quad 36$

$12 \quad 22$

$13 \quad 37$

$14 \quad 36$

RA

$16 \quad 13$

178

OA

18

$19 \quad 16$

$20 \quad 17$
$13 \times$ Stenotrophomonas maltophilia, $4 \times$ Shigella species, $3 \times \gamma$ proteobacterium, $3 \times$ Shigella sonnei, $1 \times$ Aranicola species, $1 \times$ Alcaligenes faecalis, $1 \times$ Aeromonas species, $1 \times$ Burkholderia species, $1 \times$ Bradyrhizobium elkanii, $1 \times$ Comamonas testosteroni, $1 \times$ Capnocytophaga sputigena $1 \times$ Enterococcus faecium, $1 \times$ Escherichia species, $1 \times$ Enterobacter hormaechei, $1 \times$ Pseudomonas poae, $1 \times$ Pantoea ananatis, $1 \times$ Paenibacillus humicus, $1 \times$ Rhodococcus species, $1 \times$ Ralstonia species, 1 uncultured bacterium, $1 \times$ uncultured $\gamma$ proteobacterium, $1 \times$ uncultured Flavobacterium species.

$6 \times$ Stenotrophomonas maltophilia, $5 \times$ Shigella species, $4 \times \gamma$ proteobacterium, $4 \times$ Ralstonia species, $3 \times$ Escherichia coli, $2 \times$ Bradyrhizobium elkanii, $2 \times$ Comamonas testosteroni, $2 \times$ uncultured bacterium, $1 \times$ Achromobacter xylosoxidans, $1 \times$ Alcaligenes faecalis, $1 \times$ Bacteroidetes bacterium, $1 \times$ Flavobacterium mizutaii, $1 \times$ Rhizobium radiobacter, $1 \times$ Rhodococcus species, $1 \times$ uncultured $\beta$ proteobacterium, $1 \times$ uncultured organism.

$4 \times$ Shigella species, $4 \times$ Stenotrophomonas maltophilia, $3 \times$ Alcaligenes faecalis, $2 \times$ Shigella sonnei, $2 \times$ uncultured $\beta$ proteobacterium, $2 \times$ uncultured bacterium, $1 \times$ Aminobacter aminovorans, $1 \times$ Achromobacter xylosoxidans, $1 \times$ Paenibacillus humicus, 1 $\times$ Ralstonia species, $1 \times$ uncultured organism.

$12 \times$ Stenotrophomonas maltophilia, $3 \times$ Shigella species, $3 \times$ uncultured bacterium, $2 \times$ Bacillus niacini, $2 \times$ Ralstonia species, $1 \times \beta$ proteobacterium, $1 \times$ Bradyrhizobium japonicum, $1 \times$ Burkholderia cepacia, $1 \times$ Corynebacterium durum, $1 \times$ Comamonas testosteroni, $1 \times$ Flavobacterium mizutaii, $1 \times \gamma$ proteobacterium, $1 \times$ uncultured $\beta$ proteobacterium, $1 \times$ uncultured Sphingobacterium species, $1 \times$ Mycobacterium aubagnense, $1 \times$ Shigella sonnei., $1 \times$ Sphingomonas species, $1 \times$ Sphingomonas species, $1 \times$ Pseudomonas poae, $1 \times$ Pantoea ananatis.

$10 \times$ Stenotrophomonas maltophilia, $7 \times$ Ralstonia species, $3 \times$ Comamonas testosteroni $3 \times$ uncultured bacterium, $2 \times \gamma$ proteobacterium, $1 \times$ Escherichia species, $1 \times$ Flavobacterium mizutaii, $1 \times$ Methylomicrobium species, $1 \times$ Paenibacillus humicus, $1 \times$ Pantoea ananatis, $1 \times$ Photorhabdus luminescens, $1 \times$ Streptococcus pneumoniae, $1 \times$ uncultured Sphingobacterium species, $1 \times$ uncultured Flavobacterium species, $1 \times$ uncultured $\beta$ proteobacterium, $1 \times$ uncultured Firmicutes bacterium.
$10 \times$ Stenotrophomonas maltophilia, $3 \times$ uncultured Flavobacterium species, $2 \times$ Comamonas testosteroni, $2 \times$ uncultured bacterium, $1 \times$ Aranicola species, $1 \times$ Flavobacterium mizutaii, $1 \times$ Paenibacillus humicus, $1 \times$ Shigella species, $1 \times$ Staphylococcus cohnii, $1 \times$ Sphingobacterium thalpophilum, $1 \times$ uncultured Sphingobacterium species.

$5 \times$ uncultured $\beta$ proteobacterium, $4 \times$ uncultured Flavobacterium species, $2 \times$ uncultured Sphingobacterium species, $1 \times$ Comamonas testosterone, $1 \times$ uncultured bacterium.

$3 \times$ Escherichia species, $2 \times$ uncultured bacterium, $1 \times$ Alcaligenes faecalis, $1 \times$ Comamonas testosteroni, $1 \times$ Shigella species.
$4 \times$ Stenotrophomonas maltophilia, $4 \times$ Shigella species, $2 \times$ Flavobacterium mizutaii, $2 \times$ uncultured Flavobacterium species, $2 \times$ uncultured Sphingobacterium species, $1 \times$ Acinetobacter junii, $1 \times$ Escherichia coli 0157, $2 \times$ uncultured bacterium.

$5 \times$ Stenotrophomonas maltophilia, $3 \times$ Shigella species, $3 \times$ uncultured bacterium, $1 \times$ Alcaligenes faecalis, $1 \times$ Comamonas testosteroni, $1 \times$ Flavobacterium mizutaii, $1 \times$ Paenibacillus humicus, $1 \times$ Ralstonia species.

$4 \times$ Stenotrophomonas maltophilia, $3 \times$ Shigella species, $3 \times$ uncultured bacterium, $1 \times$ Achromobacter xylosoxidans, $1 \times$ Alcaligenes faecalis, $1 \times$ Bacillus cereus, $1 \times$ Escherichia species, $1 \times$ Rothia mucilaginosa, $2 \times$ uncultured $\beta$ proteobacterium

$\mathrm{OA}=$ osteoarthritis; $\mathrm{RA}=$ rheumatoid arthritis; $\mathrm{Re} A=$ reactive arthritis; $\mathrm{UA}=$ undifferentiated arthritis.

bacterial DNA sequences have not been previously characterized by rDNA sequencing because they exhibit less than $97 \%$ similarity to known database sequences. We could find no clear association between the presence of a particular bacterial DNA and clinical symptoms.

\section{Discussion}

In the present study we used broad-range PCR amplification, cloning, and sequencing of the full-length $16 \mathrm{~S}$ rDNA from a wide variety of bacterial species in the SF samples of all patients studied. Only a few studies have been conducted to detect and identify the bacterial DNA communities in SF samples of patients with ReA, UA, and others arthropathies. In these studies, only short fragments of DNA were amplified 
Table 3

Bacterial species identified by sequencing of cloned 165 rDNA

\begin{tabular}{|c|c|c|c|c|}
\hline $\begin{array}{l}\text { Bacterium-derived DNA identified in } \\
\text { SF samples }\end{array}$ & $\begin{array}{l}\text { Number of patients in whom bacterial } \\
\text { DNAs were detected }\end{array}$ & Accession number ${ }^{\mathrm{a}}$ & Length of the sequence ${ }^{b}$ & $\%$ Similarity ${ }^{\mathrm{C}}$ \\
\hline \multicolumn{5}{|c|}{ Bacteria identified in ReA and UA patients $(n=69)$} \\
\hline \multicolumn{5}{|c|}{ Bacteria previously detected in arthritis } \\
\hline Aeromonas species & $(1 \cup A)$ & [EMBL:AF099027] & 1400 & 97.77 \\
\hline Burkholderia species & $(1 \operatorname{Re} A+1 U A)$ & [EMBL:AY769903] & 1391 & 99.13 \\
\hline Burkholderia species & $(1 \cup A)$ & [EMBL:AY134849] & 1388 & 99.71 \\
\hline Burkholderia cepacia & $(1 \mathrm{UA})$ & [EMBL:AY946010] & 1390 & 99.89 \\
\hline Escherichia coli & $(1 \operatorname{Re} A)$ & [EMBL:CP000243] & 1400 & 99.14 \\
\hline Escherichia coli & $(2 \operatorname{Re} A)$ & [EMBL:U00096] & 1400 & 100.00 \\
\hline Escherichia coli & $(1 \mathrm{Re} A+2 \mathrm{UA})$ & [EMBL:V00348] & 1400 & 99.50 \\
\hline Methylomicrobium species & $(1 \cup A)$ & [EMBL:AF194538] & 1380 & 98.83 \\
\hline Propionibacterium acnes & $(5 \operatorname{Re} A+1 U A)$ & [EMBL:AB108477] & 1386 & 99.05 \\
\hline Pseudomonas species & $(1 \mathrm{ReA})$ & [EMBL:DQ213044] & 1386 & 99.06 \\
\hline Pseudomonas species & $(1 \mathrm{Re} A)$ & [EMBL:AY014811] & 1395 & 99.06 \\
\hline Pseudomonas species & $(1 \mathrm{UA})$ & [EMBL:AM409368] & 1396 & 99.21 \\
\hline Paracoccus species & $(1 \cup A)$ & [EMBL:AY745834] & 1320 & 98.92 \\
\hline Rhodococcus species & $(2 \cup A)$ & [EMBL:AF420412] & 1374 & 97.29 \\
\hline Shigella sonnei & $(5 \mathrm{ReA}+5 \mathrm{UA})$ & [EMBL:CP000038] & 1400 & 99.71 \\
\hline Shigella sonnei & $(1 \cup A)$ & [EMBL:X96964] & 1389 & 99.78 \\
\hline Sphingomonas species & $(1 \cup A)$ & [EMBL:AB110635] & 1341 & 99.85 \\
\hline Sphingomonas species & $(1 \cup A)$ & [EMBL:AF385529] & 1341 & 100.00 \\
\hline Streptococcus species & $(1 \cup A)$ & [EMBL:AF316593] & 1400 & 98.55 \\
\hline Streptococcus species & $(1 \cup A)$ & [EMBL:AF385523] & 1400 & 99.28 \\
\hline Streptococcus mitis & $(1 \mathrm{ReA})$ & [EMBL:AJ295848] & 1400 & 97.92 \\
\hline Streptococcus mitis & $(1 \mathrm{Re} A)$ & [EMBL:AJ617805] & 1322 & 98.71 \\
\hline Streptococcus mitis & $(1 \mathrm{ReA})$ & [EMBL:AF003929] & 1396 & 99.36 \\
\hline Streptococcus mitis & $(1 \mathrm{Re} A+1 \mathrm{UA})$ & [EMBL:AY518677] & 1404 & 99.86 \\
\hline Streptococcus pneumoniae & $(2 \cup A)$ & [EMBL:AM157442] & 1400 & 98.64 \\
\hline Streptococcus thermophilus & $(2 \cup A)$ & [EMBL:AY188354] & 1397 & 99.57 \\
\hline \multicolumn{5}{|c|}{ Bacteria not previously detected in arthritis but detected in human infection } \\
\hline Agrobacterium species & $(2 \operatorname{Re} A+1 U A)$ & [EMBL:AY775177] & 1344 & 99.40 \\
\hline Bradyrhizobium elkanii & $(2 \operatorname{ReA}+4 U A)$ & [EMBL:AY904749] & 1347 & 99.40 \\
\hline Capnocytophaga sputigena & $(1 \mathrm{UA})$ & [EMBL:AF133536] & 1378 & 98.96 \\
\hline Corynebacterium durum & $(1 \cup A)$ & [EMBL:AF537593] & 1391 & 99.58 \\
\hline Delftia acidovorans & $(1 \mathrm{ReA})$ & [EMBL:AB020186] & 1309 & 97.63 \\
\hline Enterococcus faecium & $(1 \cup A)$ & [EMBL:EF533988] & 1396 & 99.42 \\
\hline Enterobacter hormaechei & $(1 \cup A)$ & [EMBL:AY995561] & 1400 & 99.70 \\
\hline Kocuria species & $(1 \cup A)$ & [EMBL:AY864652] & 1387 & 99.85 \\
\hline Mycobacterium aubagnense & $(1 \mathrm{UA})$ & [EMBL:AY859683] & 1372 & 99.27 \\
\hline
\end{tabular}


Table 3 (Continued)

Bacterial species identified by sequencing of cloned 16S rDNA

\begin{tabular}{|c|c|c|c|c|}
\hline Pantoea ananatis & $(3 \operatorname{Re} A+4 U A)$ & [EMBL:DQ133546] & 1400 & 98.80 \\
\hline Photorhabdus luminescens & $(1 \cup A)$ & [EMBL:AY444555] & 1333 & 99.85 \\
\hline Pseudomonas poae & $(2 \cup A)$ & [EMBL:AJ492829] & 1386 & 98.99 \\
\hline Rhizobium radiobacter & $(1 \cup A)$ & [EMBL:AJ389902] & 1300 & 99.67 \\
\hline Staphylococcus pasteuri & $(1 \cup A)$ & [EMBL:AJ717376] & 1411 & 99.86 \\
\hline \multicolumn{5}{|c|}{ Bacteria not previously detected in humans } \\
\hline Aquabacterium commune & $(1 \cup A)$ & [EMBL:AF035054] & 1416 & 99.70 \\
\hline Aminobacter aminovorans & $(1 \mathrm{UA})$ & [EMBL:AJ011759] & 1344 & 98.72 \\
\hline Bacillus niacini & $(1 \cup A)$ & [EMBL:AB021194] & 1400 & 99.14 \\
\hline Bradyrhizobium japonicum & $(1 \mathrm{UA})$ & [EMBL:AB072418] & 1200 & 97.81 \\
\hline Diaphorobacter species & $(1 \operatorname{Re} A)$ & [EMBL:DQ294626] & 1387 & 99.78 \\
\hline Pelomonas saccharophila & $(1 \mathrm{Re} A)$ & [EMBL:AB021407] & 1381 & 99.42 \\
\hline Paenibacillus species & $(1 \mathrm{ReA})$ & [EMBL:AM162345] & 1385 & 99.35 \\
\hline Sphingomonas faeni & $(1 \operatorname{Re} A)$ & [EMBL:AJ429239] & 1340 & 97.82 \\
\hline \multicolumn{5}{|l|}{ Uncultured bacteria } \\
\hline Bacteroidetes bacterium & $(2 U A+1 \operatorname{Re} A)$ & [EMBL:AY395022] & 1394 & $96.11^{*}$ \\
\hline Bacteroidetes bacterium & $(1 \operatorname{Re} A)$ & [EMBL:DQ195837] & 1300 & 98.39 \\
\hline$\beta$ Proteobacterium & $(2 \operatorname{Re} A+2 \cup A)$ & [EMBL:AY162033] & 1396 & 99.57 \\
\hline$\gamma$ Proteobacterium & $(4 \operatorname{Re} A+6 U A)$ & [EMBL:AY162042] & 1399 & 99.00 \\
\hline$\gamma$ Proteobacterium & $(1 \operatorname{Re} A+4 U A)$ & [EMBL:AY162068] & 1400 & 99.50 \\
\hline swine manure bacterium & $(1 \mathrm{ReA})$ & [EMBL:AY167969 ] & 1395 & 99.21 \\
\hline Uncultured $\alpha$ proteobacterium & $(1 \mathrm{ReA})$ & [EMBL:AF445680] & 1342 & 97.00 \\
\hline Uncultured Bacteroidetes bacterium & $(2 \operatorname{Re} A)$ & [EMBL:AY921801] & 1384 & 97.04 \\
\hline Uncultured bacterium & $(2 \cup A)$ & [EMBL:AY958813] & 1388 & 99.88 \\
\hline Uncultured $\beta$ proteobacterium & $(1 \operatorname{Re} A+1 U A)$ & [EMBL:AF445700] & 1372 & 99.78 \\
\hline Uncultured $\beta$ proteobacterium & $(1 \cup A)$ & [EMBL:DQ316806] & 1391 & 97.46 \\
\hline $\begin{array}{l}\text { Uncultured candidate division OP10 } \\
\text { bacterium }\end{array}$ & $(2 \cup A)$ & [EMBL:AF418946] & 1362 & $90.29^{\star}$ \\
\hline Uncultured Firmicutes bacterium & $(1 \cup A)$ & [EMBL:EF071401] & 1400 & 99.57 \\
\hline Uncultured $\gamma$ Proteobacterium & $(1 \cup A)$ & [EMBL:AF324537] & 1396 & 99.96 \\
\hline Uncultured $\gamma$ Proteobacterium & $(1 \operatorname{ReA})$ & {$[E M B L: \underline{A J 318146]}$} & 1400 & 97.63 \\
\hline Uncultured $\gamma$ Proteobacterium & $(1 \operatorname{ReA})$ & [EMBL:AY770720] & 1393 & $96.91^{*}$ \\
\hline $\begin{array}{l}\text { Uncultured Methylococcaceae } \\
\text { bacterium }\end{array}$ & $(1 \operatorname{Re} A)$ & [EMBL:EF019533] & 1398 & 97.55 \\
\hline Uncultured organism & $(2 \cup A)$ & [EMBL:DQ395839] & 1392 & 99.85 \\
\hline Uncultured soil bacterium & $(1 \operatorname{Re} A)$ & [EMBL:DQ297948] & 1340 & 99.85 \\
\hline Uncultured Streptococcus species & $(1 \cup A)$ & [EMBL:AY256519] & 1400 & 99.21 \\
\hline Uncultured Veillonella species & $(2 \cup A)$ & [EMBL:AM157449] & 1400 & 99.37 \\
\hline \multicolumn{5}{|c|}{ Bacteria identified in control group (RA and OA patients; $n=10$ ) } \\
\hline \multicolumn{5}{|c|}{ Bacteria previously detected in arthritis (in joint) } \\
\hline Acinetobacter junii & $(1 \mathrm{OA})$ & [EMBL:AB101444] & 1387 & 99.28 \\
\hline Bacillus cereus & $(1 \mathrm{OA})$ & [EMBL:AB247137] & 1399 & 98.36 \\
\hline
\end{tabular}


Table 3 (Continued)

Bacterial species identified by sequencing of cloned 16S rDNA

\begin{tabular}{|c|c|c|c|c|}
\hline Rothia mucilaginosa & $(1 \mathrm{OA})$ & [EMBL:DQ409140] & 1375 & 98.6 \\
\hline Staphylococcus cohnii & $(1 \mathrm{RA})$ & [EMBL:AJ717378] & 1399 & 99 \\
\hline \multicolumn{5}{|c|}{ Bacteria not previously detected in arthritis but detected in human infection } \\
\hline Escherichia coli 0157 & $(1 \mathrm{OA})$ & [EMBL:AE005174] & 1394 & \\
\hline \multicolumn{5}{|c|}{ Bacteria not previously detected in humans } \\
\hline Sphingobacterium thalpophilum & $(1 \mathrm{RA})$ & [EMBL:AJ43817] & 1388 & 94 \\
\hline \multicolumn{5}{|l|}{ Uncultured bacteria } \\
\hline Uncultured bacterium & $(1 \mathrm{OA})$ & [EMBL:DQ800655] & 1394 & \\
\hline Uncultured bacterium & $(1 \mathrm{RA})$ & [EMBL:AY958855] & 1388 & \\
\hline Uncultured bacterium & $(1 \mathrm{RA})$ & [EMBL:AY958896] & 1380 & \\
\hline Uncultured bacterium & $(1 \mathrm{RA}+1 \mathrm{OA})$ & [EMBL:DQ818800] & 1398 & \\
\hline
\end{tabular}

Common bacteria $^{d}(\mathrm{n}=20)$

Bacteria previously detected in arthritis

$\begin{array}{ll}\text { Achromobacter xylosoxidans } & (1 \mathrm{ReA}+2 \mathrm{UA}+1 \mathrm{OA}) \\ \text { Alcaligenes faecalis } & (2 \mathrm{ReA}+6 \mathrm{UA}+2 \mathrm{OA}+1 \mathrm{RA}) \\ \text { Comamonas testosterone } & (1 \mathrm{ReA}+4 \mathrm{UA}+1 \mathrm{OA}+3 \mathrm{RA}) \\ \text { Comamonas testosterone } & (2 \mathrm{ReA}+3 \mathrm{UA}+1 \mathrm{OA}) \\ \text { Escherichia species } & (3 \mathrm{ReA}+5 \mathrm{UA}+1 \mathrm{OA}+1 \mathrm{RA}) \\ \text { Flavobacterium mizutaii } & (4 \mathrm{ReA}+6 \mathrm{UA}+2 \mathrm{OA}+1 \mathrm{RA}) \\ \text { Shigella species } & (5 \mathrm{ReA}+8 \mathrm{UA}+3 \mathrm{OA}+2 \mathrm{RA}) \\ \text { Stenotrophomonas maltophilia } & (5 \mathrm{ReA}+8 \mathrm{UA}+3 \mathrm{OA}+1 \mathrm{RA}) \\ \text { Stenotrophomonas maltophilia } & (5 \mathrm{ReA}+8 \mathrm{UA}+3 \mathrm{OA}+1 \mathrm{RA})\end{array}$

$\begin{array}{lll}\text { [EMBL:AF439314] } & 1389 & 99.50 \\ \text { [EMBL:AY548384] } & 1395 & 99.70 \\ \text { [EMBL:AB007996] } & 1390 & 99.35 \\ \text { [EMBL:M11224] } & 1390 & 98.05 \\ \text { [EMBL:DQ337503] } & 1400 & 99.86 \\ \text { [EMBL:AJ438175] } & 1385 & 94.00 \\ \text { [EMBL:DO337523] } & 1399 & 99.70 \\ \text { [EMBL:AJ293470] } & 1396 & 99.75 \\ \text { [EMBL:AB294557] } & 1396 & 99.86\end{array}$

Bacteria not previously detected in arthritis but detected in human infection

$\begin{array}{ll}\text { Aranicola species } & (4 \mathrm{ReA}+3 \mathrm{UA}+1 \mathrm{RA}) \\ \text { Ralstonia species } & (3 \operatorname{ReA}+7 \mathrm{UA}+1 \mathrm{OA})\end{array}$

[EMBL:AM398227] 1400

99.64

Ralstonia species

[EMBL:AB045276] 1387

99.86

Bacteria not previously detected in humans

Paenibacillus humicus

$(4 \operatorname{ReA}+6 \mathrm{UA}+1 \mathrm{OA}+1 \mathrm{RA})$

[EMBL:AM411528] 1399

98.60

Uncultured bacteria

Uncultured bacterium

$(4 \operatorname{ReA}+4 \mathrm{UA}+1 \mathrm{OA})$

[EMBL:DQ818781] 1394

98.90

Uncultured bacterium

(5 $R e A+4 U A+2 O A+2 R A)$

Uncultured bacterium

$(2 \operatorname{Re} A+3 U A+1 O A+2 R A)$

[EMBL:AY838480] 1389

$94.00^{*}$

Uncultured bacterium

$(1 \operatorname{Re} A+1 \mathrm{OA})$

Uncultured bacterium

(1 UA + 1 OA)

[EMBL:AB076874] 1389

$94.00^{*}$

[EMBL:AY838458] $1382 \quad 99.64$

Uncultured $\beta$ proteobacterium

$(4 \mathrm{ReA}+6 \mathrm{UA}+1 \mathrm{OA}+1 \mathrm{RA})$

[EMBL:DQ824599] 1398

99.57

Uncultured Flavobacterium species

(3 $\operatorname{ReA}+5 \mathrm{UA}+1 \mathrm{OA}+2 \mathrm{RA})$

[EMBL:DQ366010] 1384

99.71

Uncultured Sphingobacterium

$(1 \mathrm{ReA}+3 \mathrm{UA}+1 \mathrm{OA}+2 \mathrm{RA})$

[EMBL:DQ366085] 1300

97.20

[EMBL:AB076874] 1389

Number in brackets after species names indicate the number of patient set from whom bacteria were detected. aAccession number of the bacterial species in the EMBL database. bLength of alignment on which the 16S rDNA inserted sequence and the corresponding sequence in the database are similar. 'In the ' $\%$ similarity' column, asterisks indicate highlight instances where the $\%$ similarity is below $97 \%$. ${ }^{d T h e}$ 'Common bacteria' row shows the bacteria identified in ReA, UA, RA, and OA patients.

Bacterial species detected only in SF samples and not in ST samples from our previous study [15], are indicated in bold.

$\mathrm{OA}=$ osteoarthritis; $\mathrm{RA}=$ rheumatoid arthritis; $\mathrm{ReA}=$ reactive arthritis; $\mathrm{SF}=$ synovial fluid; $\mathrm{UA}=$ undifferentiated arthritis. 
allowing solely the determination of the bacterial genus but not the identification of the species level. This is, to our knowledge, the first study using the full-length 16S rRNA gene as a target for broad-spectrum PCR to detect bacterial DNA in SF samples allowing the identification of the species level.

Sequence analysis of the PCR-positive samples revealed the presence of a wide spectrum of bacterial DNA in SF samples of all studied patients. False positivity due to contamination poses a problem when broad-range PCR targeting the $16 \mathrm{~S}$ rRNA is used [20-22]. However, our recently published study outlines extensive measures, which were also taken in the present study in order to avoid any contamination [15]. From a practical point of view, it is important to notice that we did not surgically incise the skin at the site of the aspiration thus avoiding contamination by the skin flora but our results are rather similar to those obtained by others who took this precaution [14]. PCR and extraction controls consistently yielded negative results indicating that the PCR products detected in positive samples are most likely derived from the bacterial rRNA genes actually present in SF cells. In addition, the sequences obtained varied between patients and DNA from several organisms has also been identified in arthritic human joints in independent laboratories [12-14,17,18], suggesting that their presence is unlikely to be a consequence of contamination.

The most common sequences of species found in SF samples of all patients (e.g. A. xylosoxidans, A. faecalis, F. mizutaii, and S. maltophilia) were also seen in the previous study [15], except the DNA from Aranicola species implying that they might be opportunistic colonizers of inflamed joints. $S$. maltophilia DNA was identified most frequently. This organism is an opportunistic pathogen and has previously been detected in arthritic knee joints $[12,15,17]$. Of note, other bacterial DNA have been described in human infections but not so far in arthritis were identified in the SF samples of our arthritic patients. Some of them are detected only in SF samples but not in their matched ST samples, such as $R$. radiobacter and Pantoae ananatis. Recently, we reported that DNA derived from uncultured bacteria and from environmental organisms that have not been previously detected in human samples could also be demonstrated in ST samples [15]. Similarly, in SF samples such bacterial DNA was detected including Aminobacter aminovorans, B. niacini, Diaphorobacter species, and uncultured candidate division OP10 bacterium. Several studies have also detected unsuspected uncultured and/or cultured bacteria not considered as human pathogens in arthritic joints but they were found by sequencing short DNA fragments [12-14,16]. Thus, the identification of such bacterial species after cloning and near-full length 16S DNA sequencing might be of interest and should be pursued. However, their presence in the joint can not provide definite evidence of their replication or a functional role in arthritis.
Our results also confirmed the presence of $E$. coli sequences in SF samples as previously found in ST samples of arthritis patients [15]. This could indicate the ability of $E$. coli DNA to colonize inflamed joints; the gut in different patients would be expected to contain a material derived from a range of $E$. coli 'subspecies' [23-25].

Our analysis of SF samples and their matched ST samples confirmed a wide spectrum of bacterial DNA-related species detected in each individual patient. Accordingly, a significant correlation was found between the diversity of bacterial species detected in SF and matched ST at the patient level $(r=$ $0.522, P=0.018)$. However, they revealed a different profile in regard to their known potential of triggering $\mathrm{ReA}$ in either SF or ST samples. Thus, the Shigella flexneri sequences were not detected in any of the SF samples whereas S. sonnei 16 rDNA sequences were detected more frequently in SF samples of five $\operatorname{ReA}$ and five UA patients as compared with the ST samples of one $\operatorname{ReA}$ and one UA patient [15]. DNA of Shigella species was also prevalent in SF samples as demonstrated previously in ST samples [15], but it was more frequently detected in SF samples (five ReA, eight UA, two RA, and three OA in SF vs two ReA, four UA, two RA, and one OA in ST samples). Although S. sonnei and Shigella species were detected more frequently in SF than in ST samples, the difference was statistically not significant.

We detected DNA from Shigella species in our cohort of patients with various forms of arthritis and $S$. sonnei, known to trigger ReA, only in ReA and UA samples. Shigella DNA positive patients had no clinical signs of previous intestinal infection with an enteric organism. These patients may have been asymptomatic, or the preceding gastrointestinal symptoms may have been mild and overlooked by the patients [3]. It is possible that enteric organisms may migrate from asymptomatic primary sites of the infection to the synovial compartment [3]. Most Shigella ReA caused by S. sonnei are sporadic cases [26]. The most recent published case of $S$. sonnei related $\operatorname{ReA}$ was attributed to sexual transmission of the pathogen [27]. In our study, we detected S. sonnei DNA in five ReA patients presenting with an urogenital infection, which is consistent with the possibility that this species could be related to sexual transmission.

A composition of a mixture of bacterial nucleic acids was common in our cohort of patients with various forms of arthritis, as has been described in previous studies [13-15,17,18]. The bacterial DNA might be incorporated by macrophages, which are disseminated by the circulation and reach the joint due to an increased cellular recruitment. As opposed to a single organism, such mixtures may increase the risk of triggering an immune response finally culminating in synovitis. However, genetic susceptibility factors of the host are also playing an important role particularly in persistent infections $[10,28]$. As mixtures of bacterial nucleic acids are also detectable in the 
SF samples from patients with RA and OA, we cannot exclude that this may represent a normal 'background' phenomenon not necessarily causing synovitis $[10,17]$. Another limitation of our study lies in the fact that we could not detect Chlamydia trachomatis DNA or other common bacteria known to trigger posturethritic arthritis.

\section{Conclusions}

Our study provides a valuable overall picture of the bacterial DNA environment present in the SF of the actively inflamed joints of arthritis patients. Characterization of the DNA reveals a wide spectrum of organisms so far not known to be present in human infections, not known to be present in inflamed joints of arthritis patients, and not known to trigger ReA. There is also a differential bacterial colonisation and/or infection of SF and ST samples because the analysis of SF can identify a number of bacterial DNA-related species, which have not been detected in ST samples as studied earlier [15] and has helped to confirm that the composition of bacterial DNA may change over time in joint cavity.

Accordingly, the analysis of SF or ST samples from different arthropathies patients by broad-range PCR is essentially capable of characterising the bacterial DNA environment present in joint cavity. As synovial biopsy is a difficult act, SF is well practical for such purpose.

\section{Competing interests}

The authors declare that they have no competing interests.

\section{Authors' contributions}

MS performed the experimental work, analyzed the data, and wrote the manuscript. RG conceived of the study, performed the design and coordination of the study, analyzed the data, and revised the manuscript. HF, MY, SB, NB, and SS made pathological diagnosis, conducted sampling procedures, and performed clinical and rheumatological data analyses. BJ and JS participated in the design and coordination of the study, and drafted the manuscript. MR has assisted in writing the manuscript. $\mathrm{AH}$ and $\mathrm{AS}$ analyzed microbiological and sequencing data, and revised the manuscript. All authors read and approved the final manuscript.

\section{Acknowledgements}

We thank Sebastien Chaussonneri and Sonda Guermazi (CEA-Genoscope) for help with sequence analysis and for technical assistance. We also thank llhem Cheour (Tunis), Nihel Meddeb (Tunis), Mohamed Moalla (Tunis), and Imed kolsi (Sfax) for providing patient synovial samples. This project was supported by grants from the Ministry of research and development of Tunisia with participation of funds from CEA-Genoscope-Evry-France.

\section{References}

1. Hamdulay S, Glynne SJ, Keat A: When is reactive arthritis? Postgrad Med J 2006, 82:446-453.
2. Rihl M, Klos A, Kuipers JG: Infection and musculoskeletal conditions: Reactive arthritis. Best Pract Res Clin Rheumatol 2006, 20:1119-1137.

3. Gaston JSH, Mark S, Lilli Crap MS: Arthritis associated with enteric infection. Best Pract Res Clin Rheumatol 2003, 17:219-239.

4. Gaston JSH, Cox C, Granfors K: Clinical and experimental evidence for persistent Yersinia infection in reactive arthritis. Arthritis Rheum 1999, 42:2239-2242.

5. Granfors K, Merilahti-Palo R, Luukainen R, Möttönen T, Lahesmaa $R$, Probst $P$, Märker-Hermann E, Toivanen P: Persistence of yersinia antigens in peripheral blood cells from patients with Yersinia enterocolitica 0:3 infection with or without reactive arthritis. Arthritis Rheum 1998, 41:855-862.

6. Rahman MU, Cheema MA, Schumacher HR, Hudson AP: Molecular evidence for the presence of chlamydia in the synovium of patients with Reiter's syndrome. Arthritis Rheum 1992, 35:521-529.

7. Wollenhaupt $\mathrm{J}$, Zeidler $\mathrm{H}$ : Undifferentiated arthritis and reactive arthritis. Curr Opin Rheumatol 1998, 10:306-313.

8. Hitchon CA, Peschken CA, Shaikh S, El-Gabalawy HS: Early undifferentiated arthritis. Rheum Dis Clin North Am 2005, 31:605-626.

9. Relman DA, Falkow S: Identification of uncultured microorganisms: expanding the spectrum of characterized microbial pathogens. Infect Agents Dis 1992, 1:245-253.

10. Kingsley G: Microbial DNA in the synovium: a role in aetiology or a mere bystander? Lancet 1997, 349:1038-1039.

11. Louie JS, Liebling MR: The polymerase chain reaction in infectious and post-infectious arthritis. Rheum Dis Clin North Am 1998, 24:227-236.

12. Cox CJ, Kempsell KE, Gaston JSH: Investigation of infectious agents associated with arthritis by reverse transcription PCR of bacterial rRNA. Arthritis Res Ther 2003, 5:R1-R8.

13. Kempsell KE, Cox CJ, Hurle M, Wong A, Wilkie S, Zanders ED, Gaston JS, Crowe JS: Reverse transcriptase-PCR analysis of bacterial rRNA for detection and characterization of bacterial species in arthritis synovial tissue. Infect Immun 2000, 68:6012-6026.

14. Wilbrink B, Vanderheijden IM, Schouls LM, Vanembden JDA Hazes JMW, Breedveld FC, Tak PP: Detection of bacterial DNA in joint samples from patients with undifferentiated arthritis and reactive arthritis, using polymerase chain reaction with universal 16S ribosomal RNA primers. Arthritis Rheum 1998 41:535-543.

15. Siala $M$, Jaulhac $B$, Gdoura $R$, Sibilia J, Fourati $H$, Younes $M$ Baklouti S, Bargaoui N, Sellami S, Znazen A, Barthel C, Collin E, Hammami A, Sghir A: Analysis of bacterial DNA in synovial tissue of Tunisian patients with reactive and undifferentiated arthritis by broad-range PCR, cloning and sequencing. Arthritis Res Ther 2008, 10:R40.

16. Wilkinson NZ, Kingsley GH, Jones HW, Sieper J, Braun J, Ward ME: The detection of DNA from a range of bacterial species in the joints of patients with a variety of arthritides using a nested, broad-range polymerase chain reaction. Rheumato/ogy 1999, 38:260-266.

17. Gérard HC, Wang Z, Wang GF, El-Gabalawy H, Goldbach-Mansky R, Li Y, Majeed W, Zhang H, Ngai N, Hudson AP, Schumacher HR: Chromosomal DNA from a variety of bacterial species is present in synovial tissue from patients with various forms of arthritis. Arthritis Rheum 2001, 44:1689-1697.

18. Heijden IM van der, Wilbrink B, Tchetverikov I, Schrijver IA, Schouls LM, Hazenberg MP, Breedveld FC, Tak PP: Presence of bacterial DNA and bacterial peptidoglycans in joints of patients with rheumatoid arthritis and other arthritides. Arthritis Rheum 2000, 43:593-598.

19. Cuchacovich R, Japa S, Huang WQ, Calvo A, Vega L, Vargas RB, Singh R, Flores D, Castro I, Espinoza LR: Detection of bacterial DNA in Latin American patients with reactive arthritis by polymerase chain reaction and sequencing analysis. $J$ Rheumatol 2002, 29:1426-1429.

20. Hoeffel DP, Hinrichs SH, Garvin KL: Molecular diagnostics for the detection of musculoskeletal infection. Clin Orthop Relat Res 1999, 360:37-46.

21. Kwok S, Higuchi R: Avoiding false positives with PCR. Nature 1989, 339:237-238. 
22. Rand $\mathrm{KH}$, Houck $\mathrm{H}$ : Taq polymerase contains bacterial DNA of unknown origin. Mol Cell Probes 1990, 4:445-450.

23. Melief MJ, Hoijer MA, Van Paassen HC, Hazenberg MP: Presence of bacterial flora-derived antigen in synovial tissue macrophages and dendritic cells. $\mathrm{Br} J$ Rheumatol 1995, 34:1112-1116.

24. Fredericks DN, Relman DA: Sequence-based identification of microbial pathogens: a reconsideration of Koch's postulates. Clin Microbiol Rev 1996, 9:18-33.

25. Berg RD: The indigenous gastrointestinal microflora. Trends Microbiol 1996, 4:430-435.

26. Hannu T, Mattila L, Siitonen A, Leirisalo-Repo M: Reactive arthritis attributable to Shigella infection: a clinical and epidemiological nationwide study. Ann Rheum Dis 2005, 64:594-598.

27. Chen M, Delpech V, O'Sullivan B, Donovan B: Shigella sonnei: another cause of sexually acquired reactive arthritis. Int J STD AIDS 2002, 13:135-136.

28. Colmegna I, Cuchacovich R, Espinoza LR: HLA-B27-Associated Reactive Arthritis: Pathogenetic and Clinical Considerations. Clinical Microbiology Reviews 2004, 17:348-369.

29. Wang SP, Grayston JT: Human serology in Chlamydia trachomatis infection with microimmunofluorescence. J Infect Dis 1974, 130:388-397. 